\title{
COMPUTER PREDICTION AND SYNTHESIS OF NEW OXAZOLES BASED ON AN 8-THIOSUBSTITUTED 1,3,7-TRIMETHYLXANTHINE SKELETON
}

\author{
Alexander Zlatkov ${ }^{1}$, Javor Mitkov ${ }^{2}$, Maya Georgieva ${ }^{3}$
}

\begin{abstract}
The synthesis of new oxazole derivatives was carried out under Davidson synthesis conditions from Oacylacyloins with an 8-thiosubstituted 1,3,7-trimethylxanthine skeleton and ammonium acetate in a 1:10 ratio in glacial acetic acid media. The starting O-acylacyloins were obtained as products from the interaction of the sodium salt of 2-(1,3,7trimethyl-2,6-dioxo-2,3,6,7-tetrahydro-1H-purin-8-ylthio)acetic acid and $\alpha$-haloketones. The structures of the new compounds were proven by microanalyses and spectral data. The PASS online web application was used to predict the biological activity spectra of the obtained derivatives and to determine the most promising biological effects for further experimental testing. Thus, it has been shown that the synthesized compounds are a promising class for the creation of substances with a wide range of biological activity. The substrate/metabolite specificity of the tested compounds was also predicted using SMP web-service. The studied compounds were considered to perform most probably with CYP2 substrate activity.
\end{abstract}

UDC Classification: 615.3; DOI: http://dx.doi.org/10.12955/cbup.v6.1317

Keywords: 8-thiosubstituted 1,3,7-trimethylxanthine, oxazoles, PASS online, substrate/metabolite specificity prediction

\section{Introduction:}

Oxazole, a planar heterocycle, is the parent compound for a great number of derivatives with various biological effects, found both as pharmaceuticals and drug candidates.

A number of publications are available, showing the variety of biological effects of oxazole derivatives including antifungal, antibacterial, anti-inflammatory, antiviral, antidiuretic, anticancer (Rawat et. al, 2016) and antioxidant (Rawat et al., 2016; Kus et al., 2017) activities. Oxazoles are also known for their use as diabetes II treatment, platelets aggregation inhibitor, tyrosine kinase inhibitors, and as COX-2 inhibitors (Swellmeen, 2016), as well as natural products with a proven biological activity such as marine antiviral product (-)-hennoxazole A and alkaloide pimperinine (Rawat et al., 2016). Ligands with oxazoline skeletons are also used to obtain complexes with transition metals exhibiting antibacterial activity (Shallal, 2017).

On the other hand, there are a large number of methylxanthine derivatives exhibiting extremely diverse biological effects. Their activities express as CNS stimulation, peripherial smooth muscle relaxation (broncho- and vasodilatation) and heart rate increase resultant from the antagonism to adenosine receptors and non-selective inhibition of the cyclic nucleotide phosphodiesterases (Undem et al., 2001). In addition, some 8-substituted caffeine derivatives are also known to show MAO blocking properties (Strydom et al., 2011). As demonstrated in our previous work some derivatives of 1,3,7-trimethylxanthynylthioacetic acid have expressed pronounced antihypoxic activity on different models of provoked brain hypoxia (Mitkov et al., 2007; Mitkov et al., 2010). While other 8-substituted 1,3,7-trimethylxanthines have performed great $5-\mathrm{HT} 1_{\mathrm{A}}$ and $5-\mathrm{HT} 2_{\mathrm{A}}$ receptor affinity (Valkova et al., 2012).

In recent years, in order to obtain new biologically active compounds acting on several drug targets, the idea of hybrid molecules has emerged. These include up several pharmacophore moieties with different modes of action that are covalently linked to one molecular framework. It is suggested that those compounds produced by molecular hybridization are capable of simultaneously acting on two or more conventional targets, and this multilateral targeting strategy leads to the development of a number of bioactive hybrid molecules (Ceylan et al., 2013).

The aim of the current paper is to introduce a number of newly synthesized oxazole derivatives, containing the 8-thiosubstituted 1,3,7-trimethylxanthine skeleton as modern hybrid molecules, expressing both xanthine and oxazole based biological effects.

\footnotetext{
${ }^{1}$ Alexander Zlatkov, Medical University, Faculty of Pharmacy, Department of Pharmaceutical Chemistry, Sofia, Bulgaria, alex.zlatkov@gmail.com

${ }^{2}$ Javor Mitkov, Medical University, Faculty of Pharmacy, Department of Pharmaceutical Chemistry, Sofia, Bulgaria, javor.mitkov@abv.bg

${ }^{3}$ Maya Georgieva, Medical University, Faculty of Pharmacy, Department of Pharmaceutical Chemistry, Sofia, Bulgaria,maya.bg77@gmail.com
} 


\section{Materials and methods:}

Chemistry

The materials and methods used for synthesis and proving the structure of newly synthesized compounds are described in detail in our previous publication (Georgieva et al., 2016).

\section{General method for synthesis of substituted 2-oxo-[(1,3,7-trimethyl-2,6-dioxo-2,3,6,7-tetrahydro-} 1H-purin-8-yl)sulfanyl]-acetates (2a-e, 3 and 4)

To a solution of $0.0055 \mathrm{~mol}$ of the corresponding $\alpha$-haloketone and 1-2 drops aliquot 336 in dry toluene, $0.005 \mathrm{~mol}$ of sodium salt of 2-(1,3,7-trimethyl-2,6-dioxo-2,3,6,7-tetrahydro-1H-purin-8ylthio)acetic acid was added. The reaction mixture was stirred under reflux for 2 to 14 hours. After the reaction time had elapsed (TLC monitoring), the mixture was filtered hot. The filtrate is vacuum distilled for solvent removal. The resulting crude product is recrystallized from an appropriate solvent. The compounds were obtained in a yield of $80-96 \%$. Their structure is confirmed by spectral data (FTIR, ${ }^{1} \mathrm{H}$ - and ${ }^{13} \mathrm{C}-\mathrm{NMR}$ ) and microanalysis data. The Supplementary data is available and can be provided by the authors upon request.

\section{General method for synthesis of di/tri-substituted oxazoles (5a-e, 6 and 7)}

The preparation of the di / tri substituted oxazoles was carried out under Davidson synthesis (Davidson et al., 1937). The starting compounds are mixed with ammonium acetate in a 1:10 ratio in glacial acetic acid. The reaction mixture was heated under reflux for 5 hours. The reaction medium was removed in vacuo, and the resulting crude product was washed with water and recrystallized from ethanol. The compounds were obtained in a yield of $95-98 \%$. Their structure is confirmed by spectral data (FTIR, ${ }^{1} \mathrm{H}$ - and ${ }^{13} \mathrm{C}-\mathrm{NMR}$ ) and microanalysis data. The Supplementary data is available and can be provided by the authors upon request.

\section{Results and Discussion:}

\section{Chemistry}

A number of methods have been applied for preparing di- and trisubstituted oxazoles. They may be classified into three general types, including cyclisation (Davidson oxazole synthesis and RobinsonGabriel method), functionalization and multi-component methods (Yamada et al., 2017). In all these cases, however, the reactions require strong acidic conditions and / or high temperature. Up to the present date, unfortunately no mild method for the synthesis of differently substituted oxazoles has been created.

We found, that the Davidson oxazole synthesis as the most adequate synthesis for our purposes, due to the ease in obtaining the starting compounds containing the desired methylxanthine structural fragment.

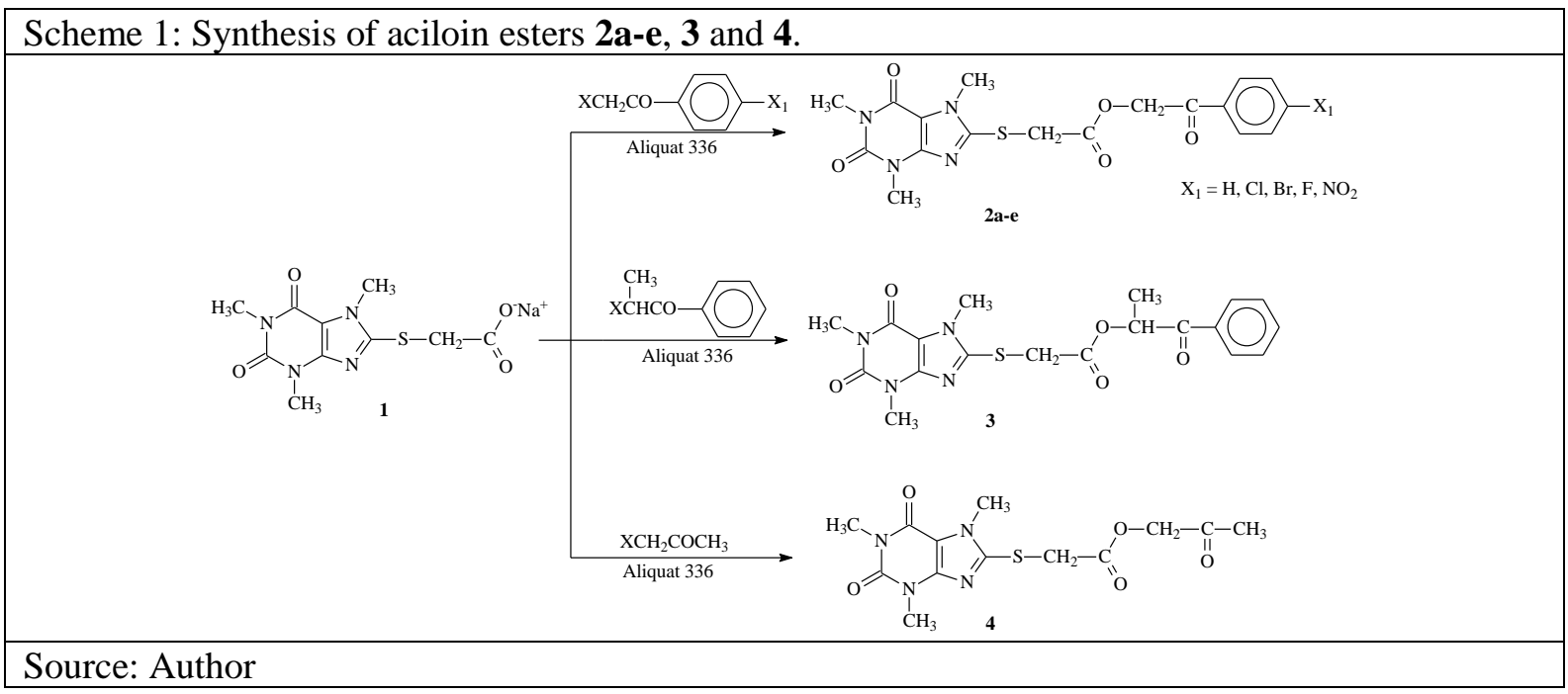

The required acyloin esters (2a-e, $\mathbf{3}$ and $\mathbf{4})$ are prepared by treatment of the corresponding $\alpha$ haloketone with the sodium salt of 2-(1,3,7-trimethyl-2,6-dioxo-2,3,6,7-tetrahydro-1H-purin-8ylthio)acetic acid ((1), also referred to as sodium 8-caffeine-thioacetate) as outlined on Scheme 1. 
The used sodium salt 1 was prepared after neutralization of 8-caffeine-thioacetate, with sodium hydroxide. The necessary 8-caffeine-thioacetate is obtained as described in (Mitkov et al., 2012). The reaction is performed under phase-transfer catalysis (solid / liquid) conditions in the presence of an aliquot 336 as a phase-transfer catalyst. The target ester products are obtained at an 80 to $96 \%$ yield. Only compound $2 \mathrm{e}$ is isolated at a $50 \%$ yield, due to problems with isolation and purification of the product. The structure of the newly synthesized acyloin esters was determined by elemental analysis and spectral data (UV, FTIR and NMR data).

The target di- / trisubstituted oxazoles 5a-e, $\mathbf{6}$ and $\mathbf{7}$ were obtained by a reaction of the esters $\mathbf{2 a - e , ~} \mathbf{3}$ and $\mathbf{4}$ with ammonium acetate in glacial acetic acid media in yields close to quantitative (Scheme 2).

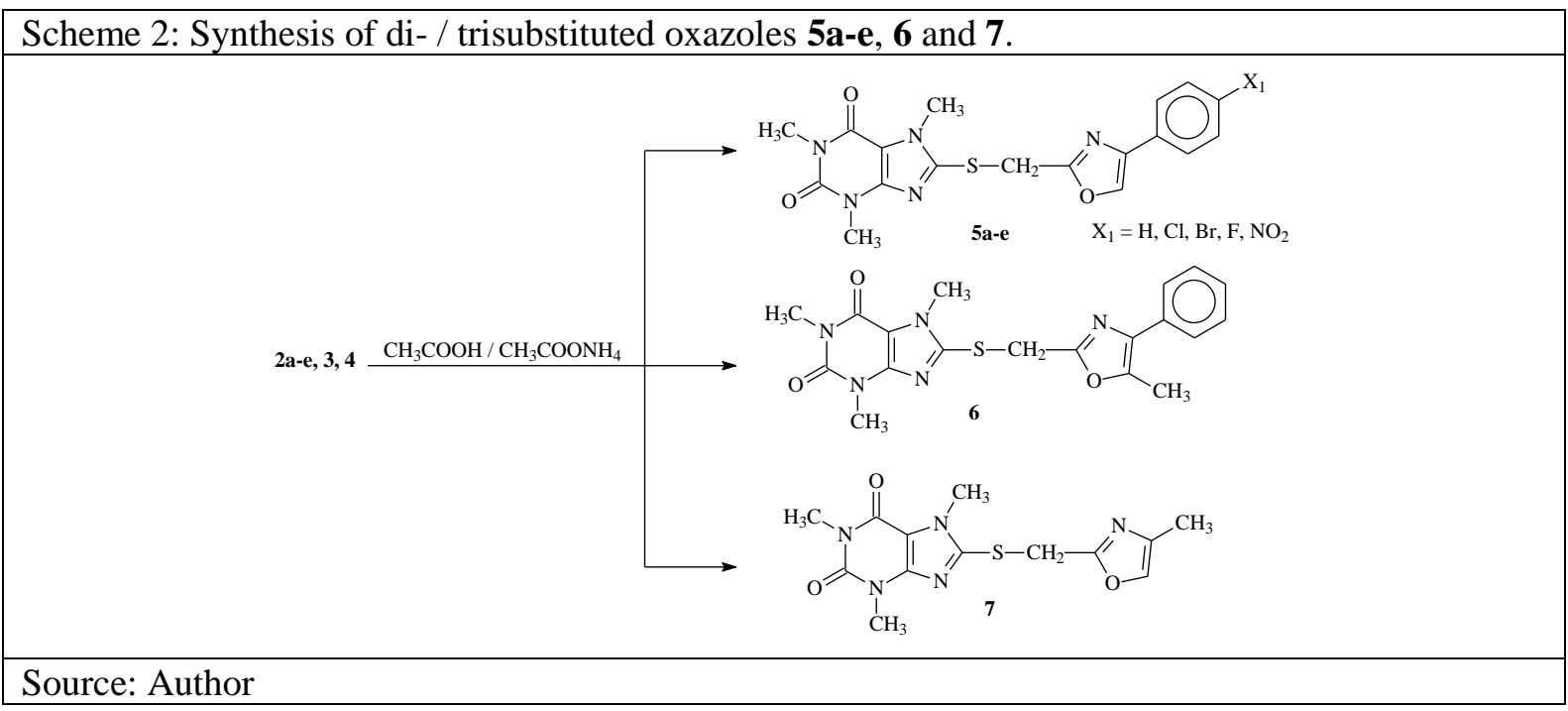

Several possible reaction pathways for reaching the oxazole ring are available in literature ( $\mathrm{Li}, 2011)$. In the present case, due to an intramolecular cyclocondensation, a formation of a protonated intermediate $\mathbf{A}$ is most possible, which after treatment with a base, upon isolation, is converted to the substituted oxazole (Scheme 3).

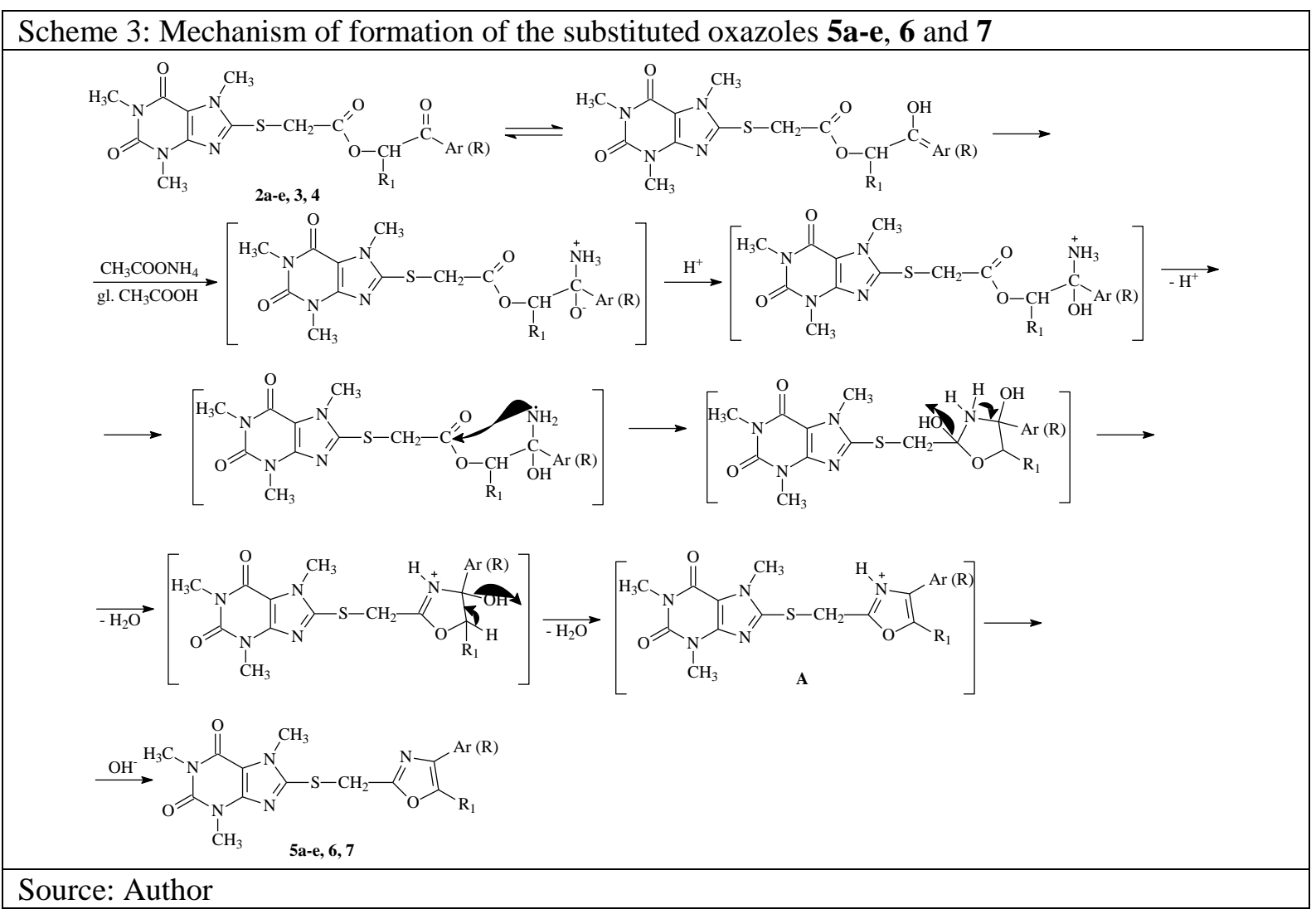




\begin{tabular}{|c|c|c|c|c|c|c|c|}
\hline Activity & $5 \mathbf{5 a}$ & $\mathbf{5 b}$ & $5 c$ & 5d & $5 e$ & 6 & 7 \\
\hline CYP2C19 inducer & 0.479 & 0.427 & 0.427 & - & 0.417 & 0.451 & 0.446 \\
\hline Cyclin-dependent kinase 9 inhibitor & 0.419 & - & 0.404 & - & - & 0.471 & 0.435 \\
\hline Nucleotide metabolism regulator & 0.426 & - & - & - & - & 0.499 & - \\
\hline CYP3A1 substrate & - & 0.480 & - & - & - & - & - \\
\hline Muscular dystrophy treatment & - & - & 0.413 & - & 0.420 & - & - \\
\hline HMGCS2 expression enhancer & - & - & 0.412 & - & - & - & - \\
\hline Kidney function stimulant & - & - & 0.473 & - & - & 0.480 & 0.528 \\
\hline Lipoprotein lipase inhibitor & - & - & - & 0.563 & - & - & - \\
\hline Cholesterol antagonist & - & - & - & 0.536 & - & - & - \\
\hline Nucleotide metabolism regulator & - & - & - & 0.499 & - & - & - \\
\hline Immunomodulator & - & - & - & 0.467 & - & - & - \\
\hline Antiulcerative & - & - & - & 0.433 & - & - & - \\
\hline Antiinflammatory & - & - & - & 0.455 & - & - & - \\
\hline CYP3A1 substrate & - & - & - & 0.423 & - & - & 0.495 \\
\hline Xanthine oxidase substrate & - & - & - & - & 0.400 & - & - \\
\hline Antidiabetic (type 2) & - & - & - & - & - & 0.456 & - \\
\hline Cyclic AMP PDE inhibitor & - & - & - & - & - & 0.451 & 0.430 \\
\hline Antineoplastic (ovarian cancer) & - & - & - & - & - & 0.400 & - \\
\hline Antidiabetic & - & - & - & - & - & 0.418 & - \\
\hline
\end{tabular}

Table 2: "Probability to be Active" (Pa) values for the predicted biological activity of 2a-e, $\mathbf{3}$ and $\mathbf{4}$.

\begin{tabular}{|l|c|c|c|c|c|c|c|}
\hline Activity & $\mathbf{2 a}$ & $\mathbf{2 b}$ & $\mathbf{2 c}$ & $\mathbf{2 d}$ & $\mathbf{2 e}$ & $\mathbf{3}$ & $\mathbf{4}$ \\
\hline Lipoprotein lipase inhibitor & 0.717 & 0.729 & 0.563 & 0.563 & 0.745 & 0.718 & 0.702 \\
\hline Cholesterol antagonist & 0.594 & 0.649 & 0.442 & 0.536 & 0.440 & 0.626 & 0.579 \\
\hline $\begin{array}{l}\text { Nucleotide metabolism } \\
\text { regulator }\end{array}$ & 0.570 & 0.526 & - & 0.499 & - & 0.491 & 0.589 \\
\hline Antiulcerative & 0.558 & 0.487 & 0.446 & 0.433 & 0.542 & & \\
\hline Cyclic AMP PDE inhibitor & 0.530 & 0.498 & 0.422 & - & 0.470 & 0.443 & 0.595 \\
\hline Immunomodulator & 0.486 & 0.440 & 0.438 & 0.467 & 0.430 & 0.424 & 0.538 \\
\hline Kidney function stimulant & 0.530 & - & 0.553 & - & - & 0.526 & 0.577 \\
\hline CYP3A1 substrate & 0.483 & 0.544 & - & 0.423 & - & 0.543 & 0.491 \\
\hline Respiratory analeptic & 0.440 & - & - & - & 0.529 & 0.484 & - \\
\hline Lipid metabolism regulator & - & 0.463 & - & - & - & 0.454 & 0.544 \\
\hline Antiinflammatory & - & 0.435 & - & 0.455 & - & - & - \\
\hline CYP2A1 substrate & - & - & - & - & 0.474 & - & - \\
\hline Calpain inhibitor & - & - & - & - & 0.419 & - & - \\
\hline $\begin{array}{l}\text { Superoxide dismutase } \\
\text { inhibitor }\end{array}$ & - & - & - & - & 0.431 & - & - \\
\hline APOA1 expression enhancer & - & - & - & - & - & 0.479 & - \\
\hline CYP2C19 inducer & - & - & - & - & - & 0.429 & 0.408 \\
\hline Analeptic & - & - & - & - & - & 0.423 & - \\
\hline $\begin{array}{l}\text { Gluconate 2-dehydrogenase } \\
\text { (accep-tor) inhibitor }\end{array}$ & - & - & - & - & - & - & 0.773 \\
\hline Vasodilator, peripheral & - & - & - & - & - & - & 0.650 \\
\hline FMO3 substrate & - & - & - & - & - & - & 0.442 \\
\hline $\begin{array}{l}\text { Flavin-containing } \\
\text { monooxygenase substrate }\end{array}$ & - & - & - & - & - & - & 0.427 \\
\hline $\begin{array}{l}\text { Note: the sign “-” is used when Pa } \\
\text { Source: Author }\end{array}$ & -0.4. & & & & & \\
\hline
\end{tabular}




\section{Computer predictions of biological activity spectra using web resource PASS online.}

The prediction of the potential biological activity spectra of the synthesized compounds was performed using the web application of the computer program PASS (Prediction of Activity Spectra for Substances) [Poroikov et al., 2002; Filimonov et al., 2014], which is freely available for the scientific community.

The PASS input information is presented as SMILES notation of the structural formulas of studied compounds. The PASS output is presented by a list of probable activities with two estimated probabilities. $P a$ - the probability to be "active", estimates the chance that the studied compound is belonging to the sub-class of active compounds (resembles the structures of molecules, which are the most typical in a sub-set of "actives" in the PASS training set). The other - Pi (the probability to be "inactive") estimates the chance that the studied compound is belonging to the sub-class of inactive compounds (resembles the structures of molecules, which are the most typical in a sub-set of "inactives" in the PASS training set).

As pharmacological effects of interest were considered, the types of activities for which the calculated $P a$ is higher than 0.4. Thus, for the analysed seven oxazoles (5a-e, $\mathbf{6}$ and 7), as well as the starting xanthine derivatives (2a-e, $\mathbf{3}$ and $\mathbf{4}$ ) the underlined effects are presented in Table $\mathbf{1}$ and Table 2, respectively.

The data obtained from the calculations using the computer on-line PASS program provided the basis for predicting the probable spectrum of pharmacological effects of a number of new xanthine esters and oxazole derivatives. The presented results in Table 1 and Table 2 identify that the predicted biological activity spectrum is wide, but still there are some literary data confirming that the selected interval of pharmacological effects is appropriate (Kus et. al, 2017; Nayana et al., 2008; Haris at al., 2005).

Additionally, the evaluated sets of compounds were subjected to a prediction of substrate/metabolite specificity, using an SMP web-service (Bezhentsev et al., 2016) for in silico prediction of the substrate/metabolite specificity. This service provides the possible interaction of the tested group of compounds with 18 cytochrome P450 and UGT isoforms: CYP1A2, CYP2C9, CYP2C19, CYP2D6, CYP3A4, UGT1A10, UGT1A1, UGT2B7, UGT1A7, UGT2B15, UGT1A8, UGT1A4, UGT2B17, UGT2B10, UGT1A3, UGT1A9, UGT1A6, UGT2B4; defined as the most probable targets. The performed prediction is based on the PASS algorithm and is defined in the software Multilevel Neighborhoods of Atoms (MNA) descriptors. The obtained data for the substrate based predictions are presented in Table 3 for compounds $2 \mathbf{a}-\mathbf{e}, \mathbf{3}, \mathbf{4}, \mathbf{5 a}-\mathbf{e}, \mathbf{6}$ and 7. The results for the corresponding metabolite based predictions for the same set of structures are presented in Table 4 .

Table 3: "Probability to be Active" (Pa) values for the substrate based prediction result of 2a-e, 3, 4,
5a-e, 6 and 7.
\begin{tabular}{|l|c|c|c|c|c|c|}
\hline & CYP2A6 & CYP2E1 & CYP2C8 & CYP1A2 & CYP1A1 & CYP2B6 \\
\hline $\mathbf{2 a}$ & 0.648 & 0.626 & 0.614 & - & - & - \\
\hline $\mathbf{2 b}$ & 0.932 & 0.900 & 0.720 & 0.846 & 0.591 & - \\
\hline $\mathbf{2 c}$ & 0.874 & 0.846 & 0.616 & 0.746 & 0.536 & - \\
\hline $\mathbf{2 d}$ & 0.608 & 0.565 & 0.616 & - & - & - \\
\hline $\mathbf{2 e}$ & 0.746 & 0.693 & 0.652 & - & 0.557 & - \\
\hline $\mathbf{3}$ & 0.632 & 0.703 & 0.679 & - & - & - \\
\hline $\mathbf{4}$ & 0.650 & 0.695 & 0.730 & 0.593 & 0.513 & - \\
\hline $\mathbf{5 a}$ & 0.908 & 0.875 & 0.715 & 0.862 & 0.597 & - \\
\hline $\mathbf{5 b}$ & 0.932 & 0.900 & 0.720 & 0.846 & 0.591 & - \\
\hline $\mathbf{5 c}$ & 0.780 & 0.776 & 0.528 & 0.605 & - & 0.552 \\
\hline $\mathbf{5 d}$ & 0.802 & 0.801 & 0.615 & 0.700 & - & - \\
\hline $\mathbf{5 e}$ & 0.896 & 0.851 & 0.650 & 0.751 & 0.621 & - \\
\hline $\mathbf{6}$ & 0.761 & 0.748 & 0.655 & 0.592 & - & - \\
\hline $\mathbf{7}$ & 0.807 & 0.788 & 0.610 & 0.706 & - & - \\
\hline
\end{tabular}
Note: the sign “-” is used when Pa < 0.5.
Source: Author


Table 4: "Probability to be Active" ( $\mathrm{Pa}$ ) values for the metabolite based prediction result of $\mathbf{2 a - e ,} \mathbf{3}$, 4, 5a-e, 6 and 7.

\begin{tabular}{|l|c|c|c|c|c|c|c|c|}
\hline & CYP2A6 & CYP2E1 & CYP1A2 & CYP3A4 & CYP2C9 & CYP2D6 & CYP2C8 & CYP1A1 \\
\hline $\mathbf{2 a}$ & & & 0.657 & 0.514 & - & - & - & - \\
\hline $\mathbf{2 b}$ & 0.551 & 0.809 & 0.928 & - & 0.720 & 0.529 & - & - \\
\hline $\mathbf{2 c}$ & - & 0.702 & 0.810 & - & 0.688 & - & - & - \\
\hline $\mathbf{2 d}$ & - & - & 0.672 & 0.628 & - & - & - & - \\
\hline $\mathbf{2 e}$ & - & 0.521 & 0.686 & 0.520 & - & - & - & - \\
\hline $\mathbf{3}$ & - & 0.556 & 0.590 & - & - & - & - & - \\
\hline $\mathbf{4}$ & - & 0.672 & 0.692 & - & 0.505 & - & - & - \\
\hline $\mathbf{5 a}$ & 0.512 & 0.807 & 0.902 & - & 0.660 & - & - & - \\
\hline $\mathbf{5 b}$ & 0.551 & 0.809 & 0.928 & - & 0.720 & 0.529 & - & - \\
\hline $\mathbf{5 c}$ & - & 0.554 & 0.693 & - & 0.607 & - & - & - \\
\hline $\mathbf{5 d}$ & - & 0.594 & 0.831 & - & 0.624 & - & - & - \\
\hline $\mathbf{5 e}$ & 0.896 & 0.851 & 0.751 & - & - & - & 0.650 & 0.621 \\
\hline $\mathbf{6}$ & - & 0.579 & 0.766 & - & 0.653 & - & - & - \\
\hline $\mathbf{7}$ & - & 0.633 & 0.794 & - & 0.546 & - & - & - \\
\hline
\end{tabular}

Note: the sign "-"” is used when $\mathrm{Pa}<0.5$.

\section{Source: Author}

Based on the obtained data presented in Tables 3 and 4, we consider the tested compounds to perform most probably with CYP2 substrate activity.

\section{Conclusion:}

New potentially biologically active substituted 2-oxo-[(1,3,7-trimethyl-2,6-dioxo-2,3,6,7-tetrahydro$1 H$-purin-8-yl)sulfanyl]acetates (2a-e, $\mathbf{3}$ and $\mathbf{4}$ ) as well as series substituted oxazoles 5a-e, $\mathbf{6}$ and $\mathbf{7}$ were obtained. The structures of these compounds have been determined, and the methods of their preparation are presented. Using the PASS online, the biological activity spectra of the synthesized compounds have been estimated. The obtained data showed that the predicted biological activity spectrum of the new xanthine esters and oxazole derivatives is wide. Further calculations determined that the tested group of compounds may be considered most probably as CYP2 substrates.

Acknowledgement. The authors would like to express their very great appreciation to prof. Vladimir Poroikov (Institute of Biomedical Chemistry, Moscow) for his valuable and constructive suggestions during the development of this research work.

\section{References}

Bezhentsev V.M., Tarasova O.A., Dmitriev A.V., Rudik A.V., Lagunin A.A., Filimonov D.A., Poroikov V.V. (2016). Computer-aided prediction of xenobiotics metabolism in the human organism. Russ. Chem. Rev., 85 (8) 854-879.

Ceylan, S.; Bektas, H.; Bayrak, H.; Demirbas, N.; Alpay-Karaoglu, S.; Ulker, S. (2013), Syntheses and biological activities of new hybrid molecules containing different heterocyclic moieties. Arch. Pharm., 346(10), 743-756.

Davidson, D.; Weiss, M.; Jelling, M. (1937), The action of ammonia on benzoin, J. Org. Chem. 2(4), 328-334.

Filimonov D.A., Lagunin A.A., Gloriozova T.A., Rudik A.V., Druzhilovskiy D.S., Pogodin P.V., Poroikov V.V. (2014). Prediction of the biological activity spectra of organic compounds using the PASS online web resource. Chemistry of Heterocyclic Compounds, 50 (3), 444-457.

Georgieva M., Mitkov J., Peikova L., Peikov P., Zlatkov Al. (2016), Synthesis evaluation and quantum mechanical characterization of cyclohexylamine containing derivatives of methylxanthine. CBU International Conference Proceedings, 4, $879-887$.

Harris P. A., Cheung M., Hunter III R. N., Brown M. L., Veal J. M., Nolte R. T., Wang L., Liu W., Crosby R. M., Johnson J. H., Epperly A. H., Kumar R., Luttrell D.K., Stafford J. A. (2005), Discovery and Evaluation of 2-Anilino-5-aryloxazoles as a Novel Class of VEGFR2 Kinase Inhibitors. J. Med. Chem. 48, 1610-1619.

Kus, C., Uğurlu, E., Ozdamar, E. D., Eke B. C. (2017), Synthesis and Antioxidant Properties of New Oxazole-5(4H)-one Derivatives. Turkish Journal of Pharmaceutical Sciences, 14(2), 174-178.

Mitkov J., Danchev N., Nikolova I., Zlatkov Al. (2007), Synthesis and brain antihypoxic activity of some aliphatic and arylaliphatic amides of caffeine-8-thioglycolic acid, Acta Pharm., 57(3), 361-370.

Mitkov J., Nikolova L., Nikolova I., Danchev N., Zlatkov Al. (2010), Synthesis and brain antihypoxic activity of some aminoalcoholic derivatives of caffeine-8-thioglycolic acid, Compt. Rend. Bulg. Acad. Sci., 63(7), 1075-1082. 
Mitkov, J., Georgieva, M., Zlatkov, Al. (2012), Development of an optimized synthetic approach for Synthesis of caffeine-8thioglycolic acid and its ester derivatives, Pharmacia, 59(1-4), 17-23.

Name Reactions in Heterocyclic Chemistry II. Ed. Jie Jack Li, (2011), John Wiley \& Sons, Inc., Hoboken, New Jersey, 221 224

Nayana M.R.S., Sekhar Y.N., Kumari N.S., Mahmood S.K., Ravikumar M. (2008). CoMFA and docking studies on triazolopyridine oxazole derivatives as p38 MAP kinase inhibitors. European Journal of Medicinal Chemistry. 43(6), 12611269.

PASSOnline retrieved from http://www.way2drug.com/PASSOnline/.

Poroikov V. V., Filimonov D. A. (2002), How to acquire new biological activities in old compounds by computer prediction, J. Comput. Aid. Molec., 16(11), 819-824.

Rawat, B. S., Shukla, S. K. (2016), Synthesis and evaluation of some new thiazole / oxazole derivatives for their biological activities. World Journal of Pharmacy and Pharmaceutical Sciences. 5 (8), 1473-1482.

Shallal, M. A. H. (2017), Synthesis, Coordination ,Chemical Analysis, Biological Activity of Ligands (Imidazole, Oxazole, thaizole) Derivatives with Zn. International Journal Of Advanced Research In Medical \& Pharmaceutical Sciences(IJARMPS), 2(8), 5-18.

SMP retrieved from http://www.way2drug.com/SMP/.

Strydom, B., Bergh, J. J., Petzer, J. P. (2011) 8-Aryl- and alkyloxycaffeine analogues as inhibitors of monoamine oxidase. European Journal of Medicinal Chemistry, 46(8), 3474-3485.

Swellmeen, L. (2016), 1,3-Oxazole Derivatives: A Review of Biological Activities as Antipathogenic. Der Pharma Chemica, 8(13), 269-286.

Undem B. J., Lichtenstein L. M: (2001), Drugs used in the treatment of asthma. In: Goodman and Gilman's the Pharmacological Basis of Therapeutics. Ed. Hardman J. G., Limbird L. E., 10th edn. Mc Graw-Hill Medical Publishing Division, International Edition, New York, 743-744.

Valkova, I., Zlatkov, Al., Nedza, K., Doytchinova, I. (2012), Synthesis, 5-HT1A and 5-HT2A receptor affinity and QSAR study of 1-benzhydryl-piperazine derivatives with xanthine moiety at N4, Med Chem Res, 21(4), 477-486.

Yamada, K., Kamimura, N., Kunishima M. (2017), Development of a method for the synthesis of 2,4,5- trisubstituted oxazoles composed of carboxylic acid, amino acid, and boronic acid. Beilstein J. Org. Chem. 13, 1478-1485. 